\title{
MicroRNA-429 inhibits gastric cancer migration and invasion through the downregulation of specificity protein 1
}

\author{
JINGBIN NI*, YISHA YANG* , DI LIU, HUI SUN, SHIMAO JIN and JINGYING LI \\ Department of Gastroenterology, Wuxi No. 2 People's Hospital Affiliated to Nanjing Medical University, \\ Wuxi, Jiangsu 214002, P.R. China
}

Received May 27, 2015; Accepted October 5, 2016

DOI: $10.3892 / \mathrm{ol} .2017 .5869$

\begin{abstract}
RNAs (miRs) have been reported to have an important role in tumorigenesis and tumor progression. Although miR-429 has been shown to be downregulated in gastric cancer (GC), the function of miR-429 in the metastasis of GC has yet to be investigated. In the present study, GC cells were transfected with miR-429, and reverse transcription-quantitative polymerase chain reaction, cell migration assays, cell invasion assays, western blot analysis and luciferase assays were conducted to investigate the role of miR-429 in GC cells. It was demonstrated that miR-429 expression was markedly increased following transfection of the cells with miR-429. Furthermore, miR-429 was shown to inhibit the migration and invasion of GC cell lines. In addition, this study provided evidence that miR-429 directly targets specificity protein 1 in GC cells. The results of the present study may enhance current knowledge regarding the molecular basis of cancer metastasis and provide a potential therapeutic strategy for GC.
\end{abstract}

\section{Introduction}

Gastric cancer (GC) is a type of malignant digestive tract tumor and is the second most common cause of cancer-associated mortality worldwide (1). It is estimated that there are 100,0000 new cases of GC and $>700,000$ GC-associated mortalities worldwide each year (2). The pathogenesis of GC is multifactorial, and genetic and epigenetic alterations of oncogenes, tumor suppressor genes and growth factors have been implicated in the development of GC (3). Treatment of GC predominantly involves surgery, chemotherapy and radiotherapy. Despite recent advances in the treatment of $\mathrm{GC},>50 \%$ of all patients

Correspondence to: Professor Jingying Li, Department of Gastroenterology, Wuxi No. 2 People's Hospital Affiliated to Nanjing Medical University, 68 Zhongshan Road, Wuxi, Jiangsu 214002, P.R. China

E-mail: jingyingli1986@163.com

*Contributed equally

Key words: gastric cancer, microRNA-429, specificity protein 1, metastasis with advanced stage GC succumb to recurrence or metastasis, and eventually death, even after a subtotal gastrectomy (4). However, currently, there exists no effective method to predict and prevent the metastasis of GC. Therefore, understanding the molecular mechanisms underlying tumor metastasis are important to further improve the survival of patients with GC. microRNAs (miRs), which are a class of small ( 22-nucleotide) non-coding RNA molecules, are widely expressed in numerous organisms (5). miRs regulate the expression of their downstream target genes by base pairing with the 3'-untranslated region (UTR) of mRNA, leading to mRNA cleavage or translation repression (6). Previous studies have verified that miRs are aberrantly expressed in various types of human cancers (7-9). Increasingly, evidence has suggested that these dysregulated miRs are critical in the proliferation, apoptosis, migration and invasion of tumor cells (10). To date, a number of dysregulated miRs have been observed in GC, and have been shown to participate in GC cell proliferation, apoptosis, migration and invasion, as well as in sensitivity to chemotherapy and radiotherapy by regulating different tumor-related target genes (11-14). Therefore, further investigation of the function of miRs will provide insight into the mechanisms of GC development and identify therapeutic targets.

miR-429 has been reported to be downregulated in GC; however, whether miR-429 has a role in the metastasis of GC has yet to be investigated. The present study aimed to elucidate the effect of miR-429 on GC motility and to investigate its underlying mechanisms.

\section{Materials and methods}

Cell culture. The human GC cell lines, SGC-7901 and AGS, were purchased from the Shanghai Institute of Biochemistry and Cell Biology (Shanghai, China). The cell lines were cultured in RPMI-1640 medium supplemented with $10 \%$ fetal bovine serum (Gibco; Thermo Fisher Scientific, Inc., Waltham, MA, USA) in an atmosphere of $5 \% \mathrm{CO}_{2}$ at $37^{\circ} \mathrm{C}$.

Cell transfection. Mature miR-429 mimics, negative control (NC) miR mimics and the luciferase reporter plasmid were designed and synthesized by Shanghai GenePharma Co., Ltd. (Shanghai, China). Cell transfection and co-transfection were performed using Lipofectamine 2000 (Invitrogen; Thermo Fisher Scientific, Inc.), according to the manufacturer's protocol. 

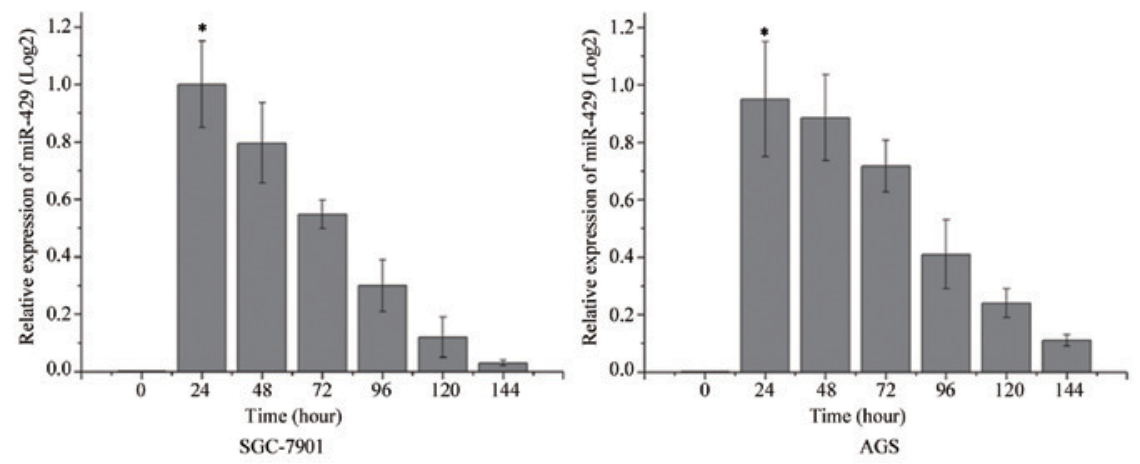

Figure 1. Expression of miR-429 in SGC-7901 and AGS gastric cancer cells prior to and following transfection with miR-429. The basal expression levels of miR-429 (0 h) in SGC-7901 and AGS cells were only at the detectable limit. The expression of miR-429 was markedly increased until $144 \mathrm{~h}$ after transfection. ${ }^{*} \mathrm{P}<0.05$. miR-429, microRNA-429.

Reverse transcription-quantitative polymerase chain reaction (RT-qPCR). Total RNA was extracted from GC cells using TRIzol reagent (Invitrogen; Thermo Fisher Scientific, Inc.), according to the manufacturer's protocol. Briefly, RNA $(500 \mathrm{ng})$ was reverse transcribed into cDNA using a reverse transcription kit (Tiangen Biotech Co., Ltd., Beijing, China). qPCR was performed on the ABI 7300 thermal cycler (Applied Biosystems; Thermo Fisher Scientific, Inc.) using SYBR PrimeScript miRNA RT-PCR kit. Every sample was analyzed three times. U6 was used as an internal control.

Cell migration and invasion assays. In vitro migration and invasion assays were performed using Transwell plates (BD Biosciences, Franklin Lakes, NJ, USA) with $8-\mu \mathrm{m}$ pores. After transfection with miR-429 or NC, the cells $\left(1 \times 10^{4}\right.$ cells) in $20 \mu \mathrm{l}$ RPMI-1640 medium were added to the upper chamber of the Transwell plates. RPMI-1640 medium containing 20\% FBS was added to the upper chamber as a chemoattractant. After 12-h incubation, cells on the upper surface were removed using cotton wool and the cells attached to the bottom were fixed with methanol and stained with $0.5 \%$ crystal violet. For the invasion assays, cells ( $1 \times 10^{4}$ cells) in $20 \mu$ l RPMI-1640 medium were added to the upper chamber pre-coated with Matrigel (BD Biosciences). After 24-h incubation, cells on the upper surface were removed using cotton wool and the cells attached to the bottom were fixed with methanol and stained with $0.5 \%$ crystal violet. Images were captured and the cells were counted using a photomicroscope (Olympus Corporation, Tokyo, Japan).

Western blot analysis. Primary antibodies used in this study included rabbit anti-human monoclonal specificity protein 1 (Sp1; dilution, 1:1,000; catalog no., 9389; Cell Signaling Technology, Inc., Beverly, MA, USA) and rabbit anti-human monoclonal $\beta$-actin (dilution, 1:1,000; catalog no., 8457; Cell Signaling Technology, Inc.). GC cells were lysed using radioimmunoprecipitation assay lysis buffer (Beyotime Institute of Biotechnology, Haimen, China) supplemented with a protease inhibitor cocktail at $72 \mathrm{~h}$ following transfection. Equal amounts of protein were separated by $10 \%$ SDS-PAGE and transferred onto polyvinylidene fluoride membranes (EMD Millipore, Billerica, MA, USA). Subsequently, the membranes were blocked with 5\% skimmed in Tris-buffered saline containing $0.1 \%$ Tween-20 at room temperature for $1 \mathrm{~h}$, and then incubated with the primary antibodies overnight at $4^{\circ} \mathrm{C}$. Goat anti-rabbit horseradish peroxidase-conjugated secondary antibody (dilution, 1:200; catalog no., 7074; Cell Signaling Technology, Inc.) was used to detect the primary antibodies. Finally, the bands were visualized using enhanced chemiluminescence reagents and images were captured using the FluorChem imaging system (ProteinSimple, San Jose, CA, USA). AlphaEase FC software (version 4.0.1; ProteinSimple, San Jose, CA, USA) was used to analyze the western blotting results.

Luciferase assay. Sp1 was identified as a target of miR-429 using TargetScan (http://www.targetscan.org/vert_71/). To determine whether $\mathrm{Sp} 1$ is a direct target of miR-429, luciferase assays were performed. Luciferase reporter plasmids, including pGL3 Sp1 3'UTR wild type (WT) and pGL3 Sp1 3'UTR mutant type (Mut), were synthesized and confirmed by GenePharma Co., Ltd. GC cells were seeded into 24-well plates at a density of $40-50 \%$ confluence and co-transfected with miR-429 or NC and the luciferase reporter plasmid using Lipofectamine 2000, according to the manufacturer's protocol. After a 48-h incubation, the cells were harvested and analyzed for luciferase activity using the Dual-Luciferase Reporter Assay System (Promega Corporation, Manheim, Germany). Each assay was replicated three times.

Statistical analysis. Data are presented as the mean \pm standard deviation, and compared using Stata 10.0 software (StataCorp LP, College Station, TX, USA). Double-tailed P-values $<0.05$ were considered to be statistically significant.

\section{Results}

Expression of miR-429 in GC cell lines prior to and following transfection with miR-429. RT-qPCR was conducted to assess the transfection efficiency following transfection of SGC-7901 and AGS cells with miR-429. As shown in Fig. 1, the basal expression of miR-429 in GC cells was at the detection limit. Following transfection of GC cells with miR-429, the expression level of miR-429 was markedly increased until $144 \mathrm{~h}$ later, as the level of miR-429 declined gradually over time.

miR-429 suppresses GC cell migration and invasion. Transwell assays were used to assess the effects of miR-429 on GC 

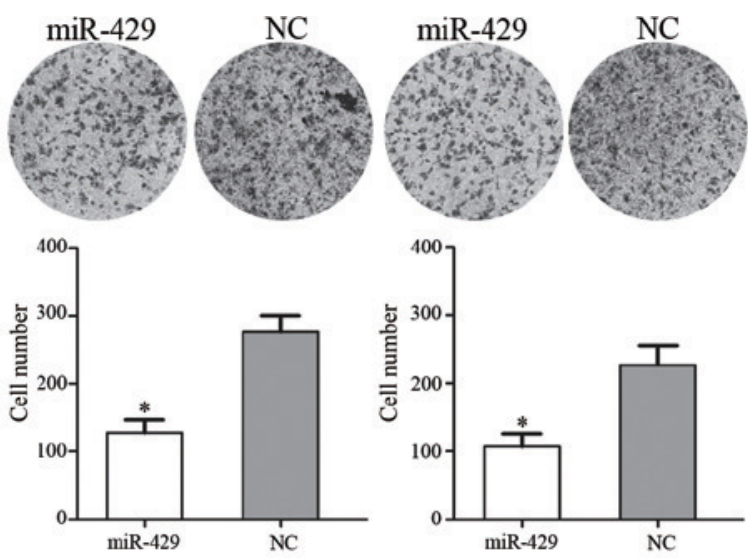

SGC-7901 migration

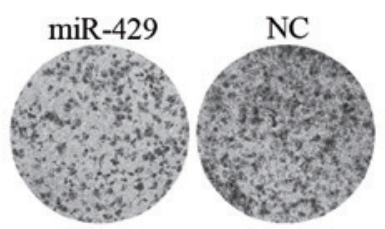

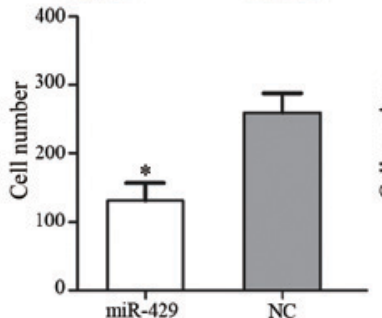

AGS migration
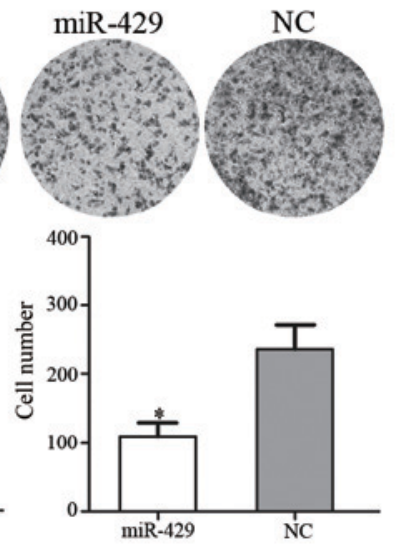

AGS invasion

Figure 2. miR-429 inhibits gastric cancer cell migration and invasion. After a 12-h incubation, the number of SGC-7901 and AGS cells that had transversed the Transwell membrane was decreased for the cells transfected with miR-429, as compared with the NC. Similarly, after 24-h of incubation, the number of SGC-7901 and AGS cells that had transversed the Transwell membrane pre-coated with Matrigel was decreased for the cells transfected with miR-429, as compared with the NC. ${ }^{*} \mathrm{P}<0.05$. miR-429, microRNA-429; NC, normal control.

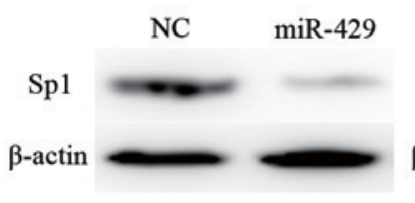

SGC-7901

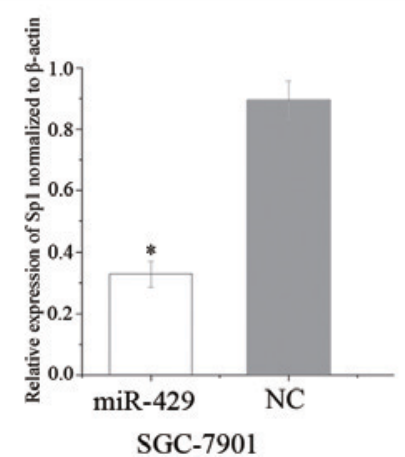

Figure 3. Western blotting and densitometric analyses demonstrated that Sp1 expression was significantly reduced in SGC-7901 and AGS gastric cancer cells following transfection of the cells with miR-429. ${ }^{*} \mathrm{P}<0.05$. Sp1, specificity protein 1; NC, normal control; miR-429, microRNA-429.
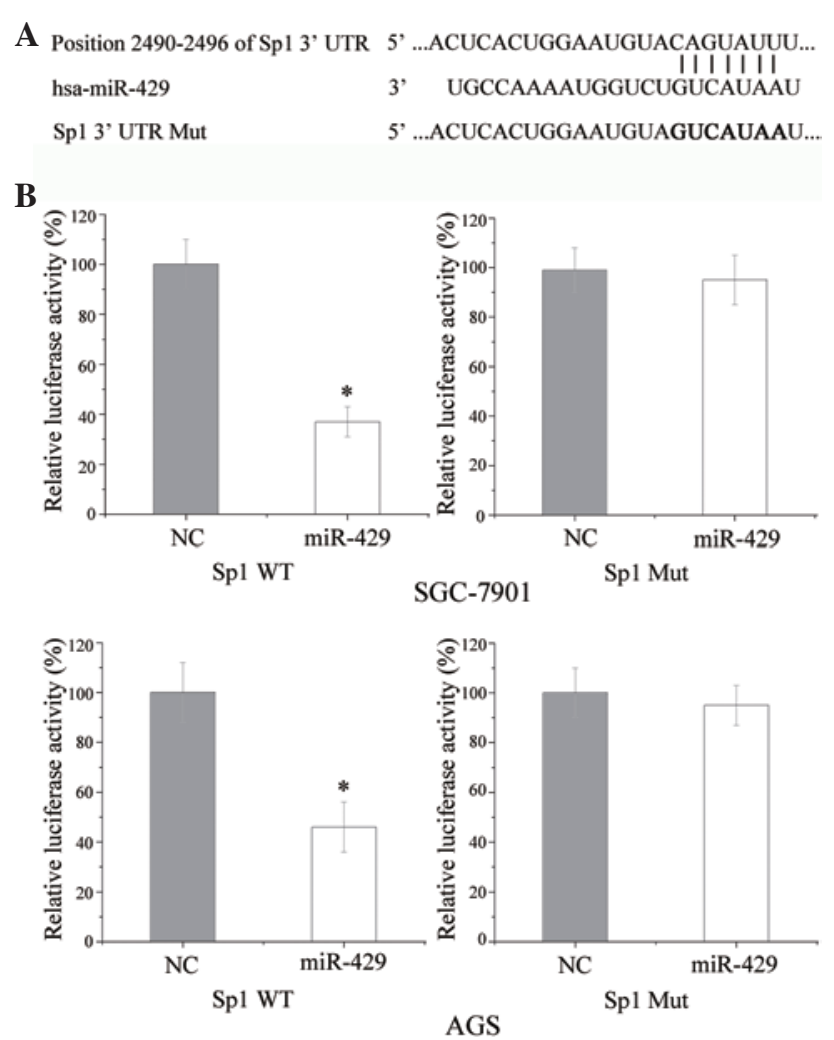

Figure 4. Sp1 is a target of miR-429. (A) TargetScan was used to demonstrate that $\mathrm{Sp} 1 \mathrm{mRNA}$ contained a miR-429 7-nucleotide seed match at position 2,490-2,496 of the Sp1 3'-UTR. (B) Luciferase activity assay verified direct binding of miR-429 to the sites of Spl predicted by TargetScan. It showed that overexpression of miR-429 significantly inhibited the WT but not the Mut luciferase activity of Sp1 in gastric cancer SGC-7901 and AGS cell lines. ${ }^{*} \mathrm{P}<0.05$. Sp1, specificity protein 1; miR-429, microRNA-429; 3'-UTR, 3'-untranslated region; WT, wild-type; Mut, mutant; NC, normal control.

SGC-7901 and AGS cell migration and invasion (Fig. 2). In the migration assay, it was demonstrated that miR-429 transfection resulted in a 53.69 $\pm 5.42 \%$ decrease in SGC-7901 cells and a $48.27 \pm 6.23 \%$ decrease in AGS cells. In the invasion assay, it was observed that miR-429 transfection led to a $56.54 \pm 5.68 \%$ decrease in SGC-7901 cells and a $52.81 \pm 7.39 \%$ decrease in AGS cells. These results suggest that miR-429 inhibits the motility of GC SGC-7901 and AGS cells.

Spl is downregulated following transfection of GC cells with $m i R-429$. Western blot analysis was performed to investigate the effect of increased expression levels of miR-429 on Sp1 expression in GC cells. As shown in Fig. 3, Sp1 was significantly downregulated in GC SGC-7901 ( $\mathrm{P}=0.021)$ and AGS $(\mathrm{P}=0.014)$ cell lines following overexpression of $\mathrm{miR}-429$. These results suggest that miR-429 reduces the protein expression level of Sp1 in GC cells.

Spl is a direct target of $m i R-429$ in GC. Sp1 was identified as a target of miR-429 using TargetScan. As shown in Fig. 4A, Sp1 mRNA contained a miR-429 7-nucleotide seed match at position 2,490-2,496 of the Sp1 3'-UTR. To verify whether miR-429 directly targets Sp1, a luciferase activity assay was performed. As shown in Fig. 4B, miR-429 significantly inhibited the luciferase activity of the vector carrying the wild-type but not the mutant Sp1 3'-UTR in GC SGC-7901 and AGS 
cells. These results suggest that Sp1 may be a direct target of miR-429 in vitro.

\section{Discussion}

The miR-200 family, which is located on chromosome 1 and has been implicated in numerous cancers, can be divided into two clusters: The first cluster consists of miR-200a, miR-200b and miR-429, and the second cluster comprises miR-200c and miR-141 (15). Previous studies have demonstrated that miR-429 is dysregulated in various cancers, including non-small cell lung cancer (16), Ehrlich ascites tumor cells (17), renal cell carcinoma (18), colorectal carcinoma, nasopharyngeal carcinoma (19) and endometrial endometrioid carcinomas (20). In addition, the expression level of miR-429 was upregulated in bladder cancer and ovarian carcinoma $(21,22)$, and its upregulation in patients with serous ovarian carcinoma was correlated with a poor prognosis (22). Therefore, miR-429 is likely involved in carcinogenesis and cancer progression, and may have different roles depending on the type of cancer.

In osteosarcoma, overexpression of miR-429 inhibited cell proliferation and enhanced cell apoptosis. Furthermore, miR-429 was shown to exert tumor-suppressing effects in osteosarcoma by binding to the 3'-UTR of zinc finger E-box binding homeobox 1 (ZEB1) (23). In esophageal carcinoma, miR-429 was shown to be downregulated and its expression was significantly associated with the occurrence of lymph node metastases. Upregulation of miR-429 inhibited cell migration and enhanced cell apoptosis in esophageal carcinoma cell lines by directly targeting B-cell lymphoma-2 and Sp1 (24). In breast cancer, downregulation of miR-429 was shown to prevent the bone metastasis of cancer cells. Conversely, ectopic expression of miR-429 markedly suppressed breast cancer cell invasion through downregulation of ZEB1 and V-Crk avian sarcoma virus CT10 oncogene homolog-like (25). Together, these findings suggested that miR-429 may used for the development of novel therapeutic strategies for cancer.

miR-429 was downregulated in human GC tissues and the level of miR-429 was associated with lymph node metastasis. Upregulation of miR-429 inhibited the proliferation of tumor cells and their attachment to fibronectin and laminin in a dose-dependent manner (26). However, no previous study has investigated the functional role of miR-429 in GC cell migration and invasion. In the present study, miR-429 was shown to inhibit cell migration and invasion by directly targeting Sp1. These findings suggested that Sp1 should be investigated as a target therapy to block GC from becoming invasive.

Sp1 is a sequence-specific DNA-binding protein that was the first transcription factor to be cloned from mammalian cells in 1983 (27). The expression level of Sp1 has been shown to be upregulated in numerous human cancers, including GC (28), breast cancer (29), hepatocellular carcinomas (30), thyroid cancer (31), colorectal cancer (32), pancreatic cancer (33) and lung cancer (34). Increasingly, evidence has suggested that Sp1 may be involved in a variety of cellular processes, including cell growth, survival, differentiation, tumor development and tumor progression (35-38). miRs, which regulate the expression of their target genes by base pairing with seed sequences in the 3'-UTR of mRNAs, have been shown to regulate the expression of Sp1 $(39,40)$. In a previous study, miR-22 was reported to be downregulated in GC and inhibited cell proliferation, migration and invasion by targeting Sp1 (41). The results of the present study suggested that miR-429 suppresses GC cell migration and invasion by directly targeting Sp1. Therefore, miR-429 could be investigated for its value in the early detection of tumor recurrence and as a potential target of therapy to prevent GC from becoming invasive.

The metastasis of GC is a key step in tumor progression and is and indicator of a poor prognosis (42). Metastasis involves a series of sequential events, including detachment, migration, invasion, extravasation, angiogenesis, survival in the circulatory system and extravasation $(43,44)$. Several miRs have been reported to have an important role in tumor metastasis (45-47). Furthermore, since miRs regulate multiple target genes simultaneously, miR-based therapy is expected to be more efficient than the traditional single target therapy $(48,49)$. In the present study, miR-429 was shown to be an important regulator in tumor cell migration and invasion, which emphasized an essential role of miR-429 in regulating GC metastasis.

In conclusion, the present study demonstrated that upregulation of miR-429 in GC cell lines inhibited GC cell metastasis in vitro. In addition, $\mathrm{Sp} 1$ was shown to be negatively regulated by miR-429 and to contain a binding site for miR-429 in the 3'-UTR of its mRNA. This newly identified association between miR-429 and Sp1 may potentially provide a novel therapeutic strategy for GC.

\section{References}

1. Siegel R, Ma J, Zou Z and Jemal A: Cancer statistics 2014. CA Cancer J Clin 64: 9-29, 2014.

2. Jemal A, Bray F, Center MM, Ferlay J, Ward E and Forman D: Global cancer statistics. CA Cancer J Clin 61: 69-90, 2011.

3. Otani K,Li X,Arakawa T, Chan FK and Yu J: Epigenetic-mediated tumor suppressor genes as diagnostic or prognostic biomarkers in gastric cancer. Expert Rev Mol Diagn 13: 445-455, 2013.

4. Kim SJ, Wang YG, Lee HW, Kang HG, La SH, Choi IJ, Irimura T, Ro JY, Bresalier RS and Chun KH: Up-regulation of neogenin-1 increases cell proliferation and motility in gastric cancer. Oncotarget 5: 3386-3398, 2014

5. Shenouda SK and Alahari SK: MicroRNA function in cancer: Oncogene or a tumor suppressor? Cancer Metastasis Rev 28: 369-378, 2009.

6. Xu L, Li Y, Yan D, He J and Liu D: MicroRNA-183 inhibits gastric cancer proliferation and invasion via directly targeting Bmi-1. Oncol Lett 8: 2345-2351, 2014.

7. Sun YC, Wang J, Guo CC, Sai K, Wang J, Chen FR, Yang QY, Chen YS, Wang J, To TS, et al: MiR-181b sensitizes glioma cells to teniposide by targeting MDM2. BMC Cancer 14: 611, 2014.

8. Chen C, Zhao Z, Liu Y and Mu D: microRNA-99a is downregulated and promotes proliferation, migration and invasion in non-small cell lung cancer A549 and H1299 cells. Oncol Lett 9: 1128-1134, 2015.

9. Zhang W, Liu K, Liu S, Ji B, Wang Y and Liu Y: MicroRNA-133a functions as a tumor suppressor by targeting IGF-1R in hepatocellular carcinoma. Tumour Biol 36: 9779-9788, 2015.

10. Lu J, Getz G, Miska EA, Alvarez-Saavedra E, Lamb J, Peck D, Sweet-Cordero A, Ebert BL, Mak RH, Ferrando AA, et al: MicroRNA expression profiles classify human cancers. Nature 435: 834-838, 2005.

11. Yang TS, Yang XH, Wang XD, Wang YL, Zhou B and Song ZS: MiR-214 regulate gastric cancer cell proliferation, migration and invasion by targeting PTEN. Cancer Cell Int 13: 68, 2013.

12. Wang F, Li T, Zhang B, Li H, Wu Q, Yang L, Nie Y, Wu K, Shi Y and Fan D: MicroRNA-19a/b regulates multidrug resistance in human gastric cancer cells by targeting PTEN. Biochem Biophys Res Commun 434: 688-694, 2013.

13. Hsu KW, Wang AM, Ping YH, Huang KH, Huang TT, Lee HC, Lo SS, Chi CW and Yeh TS: Downregulation of tumor suppressor MBP-1 by microRNA-363 in gastric carcinogenesis. Carcinogenesis 35: 208-217, 2014. 
14. Song JH and Meltzer SJ: MicroRNAs in pathogenesis, diagnosis and treatment of gastroesophageal cancers. Gastroenterology 143: $35-47$ e32, 2012

15. Tang J, Li L, Huang W, Sui C, Yang Y, Lin X, Hou G, Chen X, Fu J, Yuan S, et al: MiR-429 increases the metastatic capability of HCC via regulating classic Wnt pathway rather than epithelial-mesenchymal transition. Cancer Lett 364: 33-43, 2015

16. Zhu W, He J, Chen D, Zhang B, Xu L, Ma H, Liu X, Zhang Y and Le H: Expression of miR-29c, miR-93 and miR-429 as potential biomarkers for detection of early stage non-small lung cancer. PLoS One 9: e87780, 2014.

17. Said NA and Williams ED: Growth factors in induction of epithelial-mesenchymal transition and metastasis. Cells Tissues Organs 193: 85-97, 2011.

18. Sun Y, Shen S, Liu X, Tang H, Wang Z, Yu Z, Li X and Wu M: MiR-429 inhibits cells growth and invasion and regulates EMT-related marker genes by targeting Onecut 2 in colorectal carcinoma. Mol Cell Biochem 390: 19-30, 2014.

19. Bartis D, Mise N, Mahida RY, Eickelberg O and Thickett DR: Epithelial-mesenchymal transition in lung development and disease: Does it exist and is it important? Thorax 69: 760-765, 2014

20. Miyazono K: Transforming growth factor-beta signaling in epithelial-mesenchymal transition and progression of cancer. Proc Jpn Acad Ser B Phys Biol Sci 85: 314-323, 2009.

21. Xie P, Xu F, Cheng W, Gao J, Zhang Z, Ge J, Wei Z, Xu X and Liu Y: Infiltration related miRNAs in bladder urothelial carcinoma. J Huazhong Univ Sci Technolog Med Sci 32: 576-580, 2012.

22. Leskela S, Leandro-Garcia LJ, Mendiola M, Barriuso J, Inglada-Pérez L, Muñoz I, Martínez-Delgado B, Redondo A, de Santiago J, Robledo M, et al: The miR-200 family controls beta-tubulin III expression and is associated with paclitaxel-based treatment response and progression-free survival in ovarian cancer patients. Endocr Relat Cancer 18: 85-95, 2011.

23. Liu X, Liu Y, Wu S, Shi X, Li L, Zhao J and $\mathrm{Xu} \mathrm{H}$ Tumor-suppressing effects of miR-429 on human osteosarcoma. Cell Biochem Biophys 70: 215-224, 2014

24. Wang Y, Li M, Zang W, Ma Y, Wang N, Li P, Wang T and Zhao G: MiR-429 up-regulation induces apoptosis and suppresses invasion by targeting Bcl-2 and SP-1 in esophageal carcinoma. Cell Oncol (Dordr) 36: 385-394, 2013.

25. Ye ZB, Ma G, Zhao YH, Xiao Y, Zhan Y, Jing C, Gao K, Liu ZH and Yu SJ: miR-429 inhibits migration and invasion of breast cancer cells in vitro. Int J Oncol 46: 531-538, 2015.

26. Sun T, Wang C, Xing J and Wu D: miR-429 modulates the expression of c-myc in human gastric carcinoma cells. Eur J Cancer 47: 2552-2559, 2011.

27. Dynan WS and Tjian R: The promoter-specific transcription factor Sp1 binds to upstream sequences in the SV40 early promoter. Cell 35: 79-87, 1983.

28. Xu Y, Zhao F, Wang Z, Song Y, Luo Y, Zhang X, Jiang L, Sun Z, Miao Z and Xu H: MicroRNA-335 acts as a metastasis suppressor in gastric cancer by targeting Bcl-w and specificity protein 1. Oncogene 31: 1398-1407, 2012.

29. Yue L, Li L, Liu F, Hu N, Zhang W, Bai X, Li Y, Zhang Y, Fu L, Zhang $\mathrm{X}$ and Ye L: The oncoprotein HBXIP activates transcriptional coregulatory protein LMO4 via Sp1 to promote proliferation of breast cancer cells. Carcinogenesis 34: 927-935, 2013.

30. Yin P, Zhao C, Li Z, Mei C, Yao W, Liu Y, Li N, Qi J, Wang L, Shi Y, et al: $\mathrm{Spl}$ is involved in regulation of cystathionine gamma-lyase gene expression and biological function by PI3K/Akt pathway in human hepatocellular carcinoma cell lines. Cell Signal 24: 1229-1240, 2012.
31. Bonofiglio D, Qi H, Gabriele S, Catalano S, Aquila S, Belmonte M and Andò S: Peroxisome proliferator-activated receptor gamma inhibits follicular and anaplastic thyroid carcinoma cells growth by upregulating p21Cip1/WAF1 gene in a Sp1-dependent manner. Endocr Relat Cancer 15: 545-557, 2008.

32. Pathi S, Jutooru I, Chadalapaka G, Nair V, Lee SO and Safe S: Aspirin inhibits colon cancer cell and tumor growth and downregulates specificity protein $(\mathrm{Sp})$ transcription factors. PLoS One 7: e48208, 2012.

33. Huang C and Xie K: Crosstalk of Sp1 and Stat 3 signaling in pancreatic cancer pathogenesis. Cytokine Growth Factor Rev 23: 25-35, 2012

34. Wang YT, Chuang JY, Shen MR, Yang WB, Chang WC and Hung JJ: Sumoylation of specificity protein 1 augments its degradation by changing the localization and increasing the specificity protein 1 proteolytic process. J Mol Biol 380: 869-885, 2008.

35. Black AR, Black JD and Azizkhan-Clifford J: Sp1 and krüppel-like factor family of transcription factors in cell growth regulation and cancer. J Cell Physiol 188: 143-160, 2001

36. Li L, He S, Sun JM and Davie JR: Gene regulation by Sp1 and Sp3. Biochem Cell Biol 82: 460-471, 2004.

37. Safe $\mathrm{S}$ and Abdelrahim M: Sp transcription factor family and its role in cancer. Eur J Cancer 41: 2438-2448, 2005.

38. Wang L, Guan X, Zhang J, Jia Z, Wei D, Li Q, Yao J and Xie K: Targeted inhibition of Spl-mediated transcription for antiangiogenic therapy of metastatic human gastric cancer in orthotopic nude mouse models. Int J Oncol 33: 161-167, 2008.

39. Sun L, Liang J, Wang Q, Li Z, Du Y and Xu X: MicroRNA-137 suppresses tongue squamous carcinoma cell proliferation, migration and invasion. Cell Prolif 49: 628-635, 2016.

40. Liu X, Duan H, Zhou S, Liu Z, Wu D, Zhao T, Xu S, Yang L and Li D: microRNA-199a-3p functions as tumor suppressor by regulating glucose metabolism in testicular germ cell tumors. Mol Med Rep 14: 2311-2320, 2016.

41. Guo MM, Hu LH, Wang YQ, Chen P, Huang JG, Lu N, He JH and Liao CG: miR-22 is down-regulated in gastric cancer and its overexpression inhibits cell migration and invasion via targeting transcription factor Sp1. Med Oncol 30: 542, 2013

42. Oue N, Aung PP, Mitani Y, Kuniyasu H, Nakayama H and Yasui W: Genes involved in invasion and metastasis of gastric cancer identified by array-based hybridization and serial analysis of gene expression. Oncology 69 (Suppl 1): 17-22, 2005.

43. Gupta GP and Massague J: Cancer metastasis: Building a framework. Cell 127: 679-695, 2006

44. Klein CA: Cancer. The metastasis cascade. Science 321 1785-1787, 2008

45. Zhang W, Liu J, Qiu J, Fu X, Tang Q, Yang F, Zhao Z and Wang H: MicroRNA-382 inhibits prostate cancer cell proliferation and metastasis through targeting COUP-TFII. Oncol Rep 36: 3707-3715, 2016.

46. Zhou Q, Zhu Y, Wei X, Zhou J, Chang L, Sui H, Han Y, Piao D, Sha R and Bai Y: MiR-590-5p inhibits colorectal cancer angiogenesis and metastasis by regulating nuclear factor 90/vascular endothelial growth factor A axis. Cell Death Dis 7: e2413, 2016.

47. Cui Z and Hu Y: MicroRNA-124 suppresses Slug-mediated lung cancer metastasis. Eur Rev Med Pharmacol Sci 20: 3802-3811, 2016.

48. Shah MY, Ferrajoli A, Sood AK, Lopez-Berestein G and Calin GA: microRNA Therapeutics in Cancer - An Emerging Concept. EBioMedicine 12: 34-42, 2016.

49. Naidu S, Magee P and Garofalo M: MiRNA-based therapeutic intervention of cancer. J Hematol Oncol 8: 68, 2015. 